\title{
Predictors of referral for neoadjuvant chemotherapy prior to radical cystectomy for muscle-invasive bladder cancer and changes in practice over time
}

\author{
Geoffrey T. Gotto, MD, MPH; ${ }^{*}$ Melissa A. Shea-Budgell, MSc; ${ }^{\dagger \xi}$ M. Sarah Rose; ${ }^{; \xi}$ J. Dean Ruether, MD ${ }^{\dagger \xi}$ \\ *Department of Surgery, Cumming School of Medicine, University of Calgary, Southern Alberta Institute of Urology, Calgary, AB; †Department of Oncology, Cumming School of Medicine, University of \\ Calgary, Tom Baker Cancer Centre, Calgary, AB; §Alberta Health Services, Calgary, AB
}

Cite as: Can Urol Assoc J 2015;9(7-8):236-41. http://dx.doi.org/10.5489/cuaj.2722 Published online August 10, 2015.

\section{Abstract}

Introduction: In patients with non-metastatic muscle-invasive bladder cancer (MIBC) fit for curative therapy, a multidisciplinary approach consisting is recommended. This approach includes local treatment (usually radical cystectomy), ideally combined with neoadjuvant chemotherapy (NACT). Despite a survival benefit with NACT, uptake remains low. We assessed NACT consultation in Alberta and examined associative factors, as well as the relationship to survival.

Methods: Patients with MIBC were identified through the Alberta Cancer Registry. Demographic and clinicopathologic information was collected from electronic medical records between 2007 and 2011. In addition to descriptive statistics, logistic regression was used to determine factors associated with receiving NACT consultation. Overall survival was described using a Kaplan-Meier estimate. Results: Of the 315 radical cystectomy patients, 140 (45.1\%, 95\% confidence interval $[\mathrm{Cl}] 39.5,50.8)$ received $\mathrm{NACT}$ consultation. Patients $\geq 80$ years (odds ratio [OR] 0.21, 95\% Cl 0.08, 0.57, $p=0.002$ ) and those treated in Calgary (OR 0.11,95\% Cl 0.05, $0.25, p<0.001)$ were less likely to receive NACT consultation. The rate of NACT consultation increased steadily from 2007 to 2011 (OR 1.23, 95\% Cl 1.04, 1.45 per year of diagnosis, $p=0.018$ ). After a median follow-up of 28.1 months (range: 14.6-50.3), median survival was 54.7 months for patients who received NACT consultation versus 31.2 months for those who did not $(p=0.030)$.

Conclusions: NACT consultation in patients with MIBC undergoing radical cystectomy has improved over time; however, regional differences underscore the need for a standardized approach to NACT consultation, including common referral mechanisms.

\section{Introduction}

Urothelial carcinoma of the bladder is among the top 5 most frequently diagnosed malignancies, occurring at an age standardized rate of 16 cases per $100000 .{ }^{1}$ About 8000 Canadians were diagnosed in $2014 .^{2}$ Prognosis is in part dependent on depth of tumour invasion. ${ }^{3}$ Superficial tumours that do not invade beyond the lamina propria do well with local therapy; ${ }^{4}$ but, muscle invasive bladder cancer (MIBC) has a high rate of progression and significant risk of development of metastatic disease and death and requires more aggressive upfront multimodality treatment to achieve cure. ${ }^{5,6}$

Curative intent local treatment (i.e., radical cystectomy or external beam radiotherapy), is a viable option for many patients with clinical T2-T4 bladder cancer (53\%). ${ }^{7}$ Whenever feasible and safe, local treatment should be combined with perioperative chemotherapy to offer the best possible survival outcomes to patients. ${ }^{6}$ The strongest evidence supporting perioperative chemotherapy suggests that the neoadjuvant setting is ideal..$^{8-10}$ Provincial, national, and international guidelines for MIBC management endorse this approach. ${ }^{11-14}$ Five-year overall survival (OS) among patients with MIBC is $50 \% .^{15}$

A retrospective cohort study of 236 patients with clinical stage $\geq T 2$ bladder cancer between 2002 and 2004 treated with radical cystectomy in Edmonton evaluated the rate of referral to medical oncology for consideration of neoadjuvant chemotherapy (NACT). Following the development of a provincial guideline, the referral rate was $23.4 \%$ and the rate of receipt of NACT was $14.0 \% .^{16}$

We were interested in evaluating NACT consultation rates in this patient population in Alberta and whether rates were improving in the years following 2004 based on the Edmonton experience. We conducted a retrospective cohort study of all Alberta patients diagnosed with MIBC from 2007 through 2011 and examined the rate of consultation for consideration of NACT. We also evaluated the OS of the patients in this cohort. 


\section{Methods}

Patients diagnosed with MIBC between January 1, 2007 and December 31, 2011 were identified from the Alberta Cancer Registry. A transurethral resection of a bladder tumour indicating tumour invasion into muscularis propria followed by a cystectomy resulted in inclusion in the cohort. Patients with a bladder tumour that was not a primary urothelial carcinoma were excluded. Electronic medical records were reviewed for 315 patients. This quality improvement study was deemed minimal risk by the project ethics tool (A pRoject Ethics Community Consensus Initiative [ARECCI]). ${ }^{17}$

Our intention in examining this retrospectively collected cohort of patients was to measure uptake of the Alberta Genitourinary Tumour Team's evidence-based practice guideline on the management of MIBC, specifically regarding recommendations for NACT. ${ }^{11}$ Alberta's provincial guidelines state that patients with MIBC should be referred to a medical oncologist for consideration of NACT. The guideline was developed in 2005 and then updated regularly through the study period (2011). Demographics and clinicopathologic information (date of diagnosis, date of cystectomy, surgeon, date of NACT consultation, NACT offer/ receipt, and pathologic stage) was collected from electronic medical records. Surgeon case load was calculated as the mean number of cases per year. ${ }^{18}$

The population was described using the median and quartile range for the continuous variables and percentages of categorical variables. Demographic and clinicopathological features were compared between patients who received a medical oncology consultation and those who did not, using logistic regression clustering on surgeon code to account for intra-surgeon correlation. Similarly, a multivariable logistic regression analysis was used to determine factors associ-

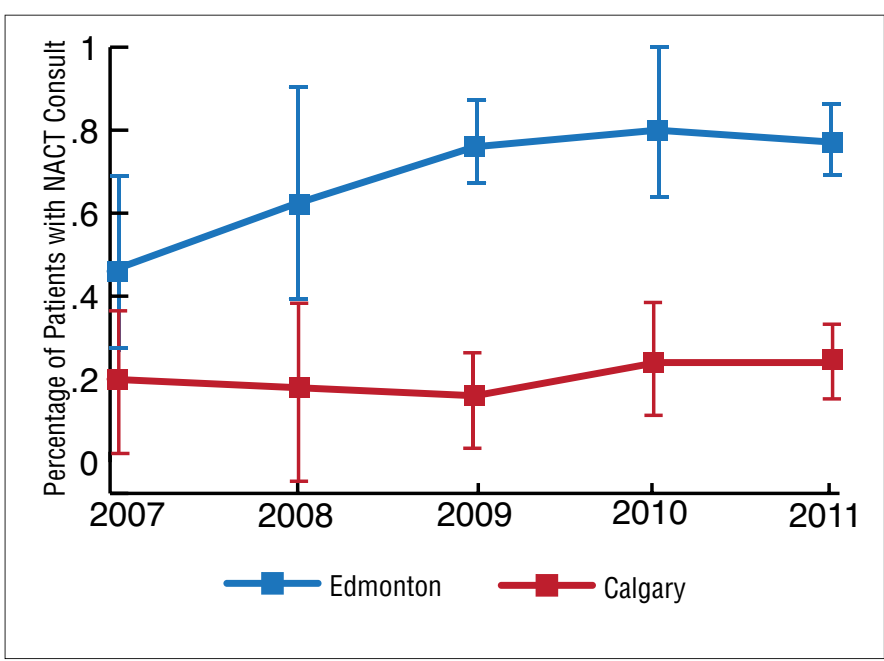

Fig. 1. Trends over time in neoadjuvant chemotherapy consultation rates for Edmonton (blue line) to Calgary (red line). ated with receiving a NACT consultation. Variables which were significant at $p<0.20$ individually were included in the model. Variables that were not significant at $p<0.05$ were removed one at a time from the full model, examining the stability of the regression coefficients to assess potential confounding, to arrive at the final model. OS was described using a Kaplan-Meier estimate. Patients still alive were censored at their last observation. Potential differences in OS due to demographic and clinicopathological variables were examined using an individual proportional hazards model clustering on surgeon code to account for intra-surgeon correlation. A multivariable model predicting OS was developed using the same procedure as for the logistic regression. All tests were two-sided, with $p<0.05$ defined as statistically significant. Stata (StataCorp LP, College Station, TX) was used to perform all statistical analyses.

\section{Results}

The median age of our cohort was 68.5 years and most $(65.7 \%)$ were between 60 and 79 years (Table 1$)$. Male patients predominated $(75.9 \%)$ and slightly less were treated in Calgary than in Edmonton ( $45.7 \%$ vs. $50.8 \%$, respectively) (Table 1). Among 315 patients with MIBC who underwent cystectomy, $140(45.1 \%, 95 \% \mathrm{Cl} 39.5,50.8)$ received a NACT consultation. The 5 -year rate of NACT consultation was $69.4 \%$ in Edmonton and $20.1 \%$ in Calgary (Table 2). Year by year, the NACT consultation rate was consistently higher in Edmonton than Calgary $(p<0.001$; Fig. 1$)$. Between 2007 and 2011, NACT consultation had increased over 1.6-fold in Edmonton from 46.2\% (95\% confidence interval $[\mathrm{Cl}] 22.2,70.1)$ to $76.7 \%(95 \% \mathrm{Cl} 67.7 \%, 85.7 \%)$. In Calgary, NACT consultation increased by 1.3 -fold from $19.2 \%(95 \% \mathrm{Cl} 1.3 \%, 37.2 \%)$ to $25.0 \%$. (95\% Cl $16.1 \%$,

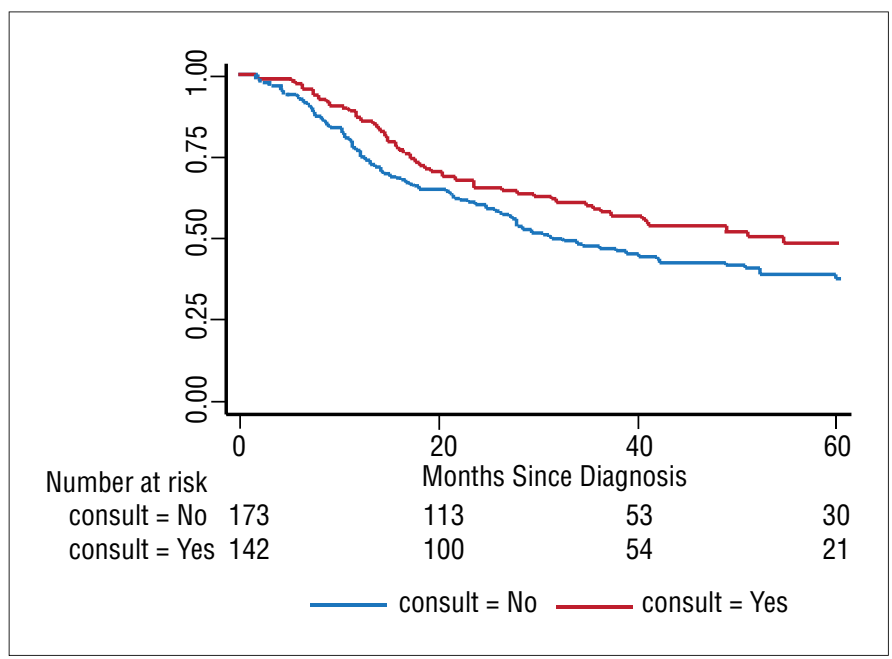

Fig. 2. Kaplan-Meier analysis of overall survival for patients who received a neoadjuvant chemotherapy consultation (red line) and those who did not (blue line). 


\begin{tabular}{|c|c|}
\hline Characteristic & $(\mathrm{N}=315)$ \\
\hline \multicolumn{2}{|l|}{ Age (years) } \\
\hline Years: median (range) & $68.5(41-91)$ \\
\hline <60 years: $n(\%)$ & $67(21.3)$ \\
\hline$>60$ to $<80$ years: $n(\%)$ & $207(65.7)$ \\
\hline$>80$ years: $n(\%)$ & $41(13.0)$ \\
\hline Male sex: n (\%) & 239 (75.9) \\
\hline \multicolumn{2}{|l|}{ Cystectomy location: $\mathrm{n}(\%)$} \\
\hline Calgary & $144(45.7)$ \\
\hline Edmonton & $160(50.8)$ \\
\hline Other & $11(3.5)$ \\
\hline \multicolumn{2}{|l|}{ Year of diagnosis } \\
\hline 2007 & $52(16.5)$ \\
\hline 2008 & $73(23.2)$ \\
\hline 2009 & $71(22.5)$ \\
\hline 2010 & $52(16.5)$ \\
\hline 2011 & $67(21.3)$ \\
\hline
\end{tabular}

$33.9 \%)$. By 2011 , the overall rate of NACT consultation was $49.3 \%$ (95\% Cl 27.9, 70.6).

NACT consultation was not influenced by patient gender ( $p=0.349$ ) or surgeon caseload ( $p>0.2$ for all categories). Patients over 80 were significantly less likely to receive a NACT consultation (odds ratio [OR] 0.21, 95\% Cl 0.08, $0.57, p=0.002$ ) as were patients who had their cystectomy in Calgary (OR 0.11, 95\% Cl 0.05, 0.25, $p<0.001$ ). The number of patients who received a NACT consultation increased steadily over the 5 years (OR $1.23,95 \% \mathrm{Cl} 1.04$, 1.45 per year of diagnosis, $p=0.018$ ) (Table 3). There was no evidence of interaction between location and year of diagnosis $(p=0.254)$. Significant predictors in the multivariable model were: patient age of $\geq 80$ (OR $0.11,95 \%$ $\mathrm{Cl} 0.05,0.24, p<0.001)$, cystectomy location in Calgary (OR 1.32, 95\% Cl 1.03, 1.70, $p=0.031$ ), and an incremental increase in the year of diagnosis (OR 1.32, 95\% Cl 1.03, $1.70 p=0.031$ ) (Table 3).

The median follow-up for the entire cohort was 28.1 months (quartile range 14.6-50.3). The median OS was 40.4 months and differed for patients who received NACT consultation versus those who did not (54.7 vs. 31.2 months) $(p=0.030)$. The 5 -year cumulative OS rates were $48.7 \%$ $(95 \% \mathrm{Cl} 38.7,58.0)$ and $37.7 \%(95 \% \mathrm{Cl} 29.6,45.8)$, respectively, for those who did and those who did not receive a consult (Fig. 2).

Individual variables that were significant predictors of OS at $p<0.20$ included NACT consult (hazard ratio [HR] 0.72, $95 \% \mathrm{Cl} 0.53,0.97, p=0.030)$, receipt of NACT (HR 0.62, $95 \% \mathrm{Cl} 0.41,0.95, p=0.026)$, pathologic stage ( $\leq \mathrm{pT} 1$ postcystectomy) (HR 3.98, 95\% Cl 2.53, 6.25, $p<0.001$ ), and patient age $(p=0.002)$ with increasing hazards as patient age increased. Compared to patients under 60 , the HR was $1.41(95 \% \mathrm{Cl} 0.81,2.44)$ for patients between 60 and 69,
Table 2. Receipt of NACT consultation, offering of NACT, and receipt of NACT (2007-2011)

\begin{tabular}{lcc}
\hline Management characteristic & $\begin{array}{c}\text { Edmonton } \\
\mathbf{N}=\mathbf{1 6 0}\end{array}$ & $\begin{array}{c}\text { Calgary } \\
\mathbf{N}=\mathbf{1 4 4}\end{array}$ \\
Received NACT consultation & $111(69.4 \%)$ & $29(20.1 \%)$ \\
Offered NACT & $97(60.6 \%)$ & $19(13.2 \%)$ \\
Received NACT & $91(56.9 \%)$ & $16(11.1 \%)$ \\
\hline NACT: neoadjuvant chemotherapy. & & \\
\hline
\end{tabular}

$1.68(95 \% \mathrm{Cl} 1.04,2.71)$ for patients between 70 and 79 , and $2.84(95 \% \mathrm{Cl} 1.67,4.83)$ for patients 80 and over. In the multivariable model, only patient age and pathologic stage remained significant predictors (Table 4). After including these two variables in the model, neither NACT consult (HR 0.95, 95\% Cl 0.70, 1.28, $p=0.716$ ) nor receipt of NACT (HR 0.86, 95\% Cl 0.58, 1.28, $p=0.449$ ) were significant predictors of OS. NACT consultation and pathologic stage post-cystectomy were highly related $(p<0.001)$. Only $12.1 \%$ of patients who did not receive NACT consultation were $\leq \mathrm{pT} 1$ compared to $36.1 \%$ of patients who did. When receipt of NACT was included without pathologic stage, it was not statistically significant (HR $0.71,95 \% \mathrm{Cl} 0.47$, $1.06, p=0.095)$.

\section{Discussion}

Within Alberta, guidelines for the management of MIBC have been developed under the direction of the provincial Tumour Team. We evaluated adherence to a recommendation supporting NACT consultation in this population, as NACT has been shown to offer survival benefit. ${ }^{19,20} \mathrm{We}$ identified 315 patients with newly diagnosed MIBC between 2007 and 2011. The overall rate of NACT consultation was $45.1 \%$. Evidence suggests that elderly ( $>80$ years) patients can tolerate platinum-based chemotherapy well and derive similar benefits to younger patients..$^{21,22}$ Not surprisingly, however, in our cohort, patients 80 and over were significantly less likely to receive NACT consultation, similar to data reported elsewhere. ${ }^{23}$ In our cohort, location of surgery was a predictive factor.

It was encouraging to see the increase in NACT consultation from $46.2 \%$ in 2007 to $76.7 \%$ in 2011 in Edmonton and from $19.2 \%$ in 2007 to $25.0 \%$ in 2011 in Calgary. In comparison, a retrospective review of data from patients in the National Cancer Data Base with no prior malignancy who ultimately underwent radical cystectomy for MIBC from 2006 to 2010 revealed an increase in the rate of NACT use from $10.1 \%$ in 2006 to $20.8 \%$ in 2010 ( $p=0.005) .{ }^{24}$ Regional differences within the province were evident, and in Calgary in 2011 the referral rate was still only $25.0 \%$ in spite of the development of provincial guidelines. Possible reasons for poor uptake in Calgary include skepticism on the part of some surgeons regarding the benefits of NACT, lack 


\begin{tabular}{|c|c|c|c|c|c|c|c|c|}
\hline \multirow[t]{2}{*}{ Variable } & \multicolumn{4}{|c|}{ Bivariable analysis } & \multicolumn{4}{|c|}{ Multivariable analysis } \\
\hline & OR & & & $p$ value & OR & & & $p$ value \\
\hline \multicolumn{9}{|l|}{ Location } \\
\hline Edmonton & 1.00 & & & & 1.00 & & & \\
\hline Calgary & 0.11 & 0.05 & 0.25 & $<0.001$ & 0.11 & 0.05 & 0.24 & $<0.001$ \\
\hline \multicolumn{9}{|c|}{ Year of diagnosis (per year increase) } \\
\hline & 1.22 & 1.04 & 1.45 & 0.018 & 1.32 & 1.03 & 1.70 & 0.031 \\
\hline \multicolumn{9}{|l|}{ Sex } \\
\hline Male & 1.00 & & & & & & & \\
\hline Female & 0.83 & 0.55 & 1.23 & 0.349 & & & & \\
\hline \multicolumn{9}{|c|}{ Annual surgeon case load } \\
\hline$\leq 1.5$ & 1.00 & & & & & & & \\
\hline $1.6-2.5$ & 0.60 & 0.19 & 1.91 & 0.388 & & & & \\
\hline $2.6-6.0$ & 1.74 & 0.64 & 4.72 & 0.277 & & & & \\
\hline$>6.0$ & 3.26 & 0.51 & 20.9 & 0.212 & & & & \\
\hline \multicolumn{9}{|l|}{ Age } \\
\hline$<80$ & 1.00 & & & & 1.00 & & & \\
\hline$\geq 80$ & 0.21 & 0.08 & 0.57 & 0.002 & 0.21 & 0.06 & 0.71 & 0.012 \\
\hline
\end{tabular}

of a formal referral pathway to medical oncology for patients with MIBC being considered for cystectomy, and anecdotal evidence of an imbalance in surgeon volumes (i.e., more surgeons are performing cystectomies in Calgary than in Edmonton), leading to greater variability in practice. Lastly, in Edmonton through the University of Alberta, there is a formal resident training program, whereas no such program exists in Calgary. Improved communication through multidisciplinary tumour boards has been shown to improve NACT referral in other malignancies (breast cancer, ${ }^{25}$ esophageal cancer $^{26}$ ), and may be a potential strategy to improve NACT consultation in MIBC. The Southern Genitourinary Tumour Group has since implemented a mechanism for identifying patients at the level of the pathologist and notifying the tumour group nurse of any new muscle-invasive cases. The nurse then adds these cases to rounds to ensure they are discussed with a medical oncologist. Anecdotally, the rates of referral since implementation of this initiative have resulted in a significant increase in the NACT consultation rate in Calgary; however, data from 2013 onward are needed to confirm any changes in the NACT consultation rates.

The median OS of our cohort was 40.4 months. The 23.5-month difference between patients who did receive a NACT consultation and those who did not was statistically significant. There was a 5-year cumulative OS difference of $11 \%$ comparing these 2 patient groups. Factors associated with improved OS included $\leq \mathrm{pT} 1$ pathology post-cystectomy and patient age $<70$. Receipt of NACT consultation and ultimately NACT were significant predictors of survival on univariate analysis, but did not remain significant on multivariate analysis. In a previously described case series from Alberta, receipt of NACT plus cystectomy versus cystectomy alone was significantly associated with tumour down staging $(56.3 \%$ vs. $25.9 \%$, respectively, $p=0.009),{ }^{16}$ similar to results reported by others. ${ }^{27,28}$

Average surgeon caseload was not associated with receipt of NACT consultation or OS. However, there was a trend towards NACT consultation and reduced mortality with increasing caseload. The odds of receiving a consultation was 3.26-fold higher (not statistically significant) among patients treated by high caseload ( $\geq 7$ cases per year) versus low caseload ( $\leq 1.5$ cases per year) surgeons. Likewise, the risk of death was $14 \%$ lower (not statistically significant) among patients treated by high caseload surgeons. A similar non-significant trend in decreased mortality was reported among 3296 patients treated with cystectomy in Ontario between 1992 and 2004. ${ }^{18}$ Most of these patients would have been treated before the creation of guidelines recommending NACT in MIBC. A series from the United Kingdom of 6308 cystectomy patients reported a significant inverse correlation $(-0.968, p<0.01)$ between caseload and mortality. ${ }^{29}$ There are no data available elsewhere on the relationship between surgeon caseload and receipt of NACT consultation among patients with MIBC. These data are interesting and suggest a possible advantage to centralizing cystectomies in Alberta to allow for more regular and coordinated multidisciplinary discussion of NACT in MIBC patients. Further study with a larger sample size to evaluate this question is warranted.

Our study has several limitations including the retrospective nature of the data. The electronic medical record was introduced in 2005 and not all data elements could be collected. For example, the surgeon on record could not be obtained in every case. We were unable to collect 
Gotto et al.

\begin{tabular}{|c|c|c|c|c|c|c|c|c|}
\hline \multirow{3}{*}{ NACT receive } & \multicolumn{4}{|c|}{ Bivariable analysis } & \multicolumn{4}{|c|}{ Multivariable analysis } \\
\hline & \multirow[t]{2}{*}{ HR } & \multicolumn{2}{|c|}{$95 \% \mathrm{Cl}$} & \multirow[t]{2}{*}{$p$ value } & \multirow[t]{2}{*}{ HR } & \multicolumn{2}{|c|}{$95 \% \mathrm{Cl}$} & \multirow[t]{2}{*}{$p$ value } \\
\hline & & & & & & & & \\
\hline No & 1.00 & & & & & & & \\
\hline Yes & 0.62 & 0.41 & 0.95 & 0.026 & & & & \\
\hline \multicolumn{9}{|l|}{ NACT consult } \\
\hline No & 1.00 & & & & & & & \\
\hline Yes & 0.72 & 0.53 & 0.97 & 0.030 & & & & \\
\hline \multicolumn{9}{|l|}{ Location } \\
\hline Edmonton & 1.00 & & & & & & & \\
\hline Calgary & 1.08 & 0.81 & 1.43 & 0.609 & & & & \\
\hline \multicolumn{9}{|c|}{ Year of diagnosis } \\
\hline Per year & 1.02 & 0.92 & 1.14 & 0.706 & & & & \\
\hline \multicolumn{9}{|c|}{ Pathologic stage post-cystectomy } \\
\hline$\leq \mathrm{pT} 1$ & 1.00 & & & & 1.00 & & & \\
\hline$\geq \mathrm{pT} 2$ & 3.98 & 2.53 & 6.25 & $<0.001$ & 3.74 & 2.43 & 5.77 & $<0.001$ \\
\hline \multicolumn{9}{|l|}{ Sex } \\
\hline Male & & 1.00 & & & & & & \\
\hline Female & 0.80 & 0.59 & 1.07 & 0.124 & & & & \\
\hline \multicolumn{9}{|c|}{ Annual surgeon case load } \\
\hline$\leq 1.5$ & 1.00 & & & 0.400 & & & & \\
\hline $1.6-2.5$ & 0.65 & 0.39 & 1.07 & & & & & \\
\hline $2.6-6.0$ & 0.82 & 0.54 & 1.24 & & & & & \\
\hline$>6.0$ & 0.86 & 0.57 & 1.30 & & & & & \\
\hline \multicolumn{9}{|l|}{ Age } \\
\hline$<60$ & 1.00 & & & 0.002 & 1.00 & & & \\
\hline $60-69$ & 1.41 & 0.81 & 2.44 & & 1.48 & 0.90 & 2.44 & 0.121 \\
\hline $70-79$ & 1.68 & 1.04 & 2.71 & & 1.58 & 1.02 & 2.44 & 0.041 \\
\hline$\geq 80$ & 2.84 & 1.67 & 4.83 & & 2.48 & 1.45 & 4.20 & 0.001 \\
\hline
\end{tabular}

information regarding the reasons for non-receipt of NACT consultation, including comorbidities (i.e., poor creatinine clearance) and patient preference. Strengths of this study include the population-based data; all new MIBC cases diagnosed in Alberta between 2007 and 2011 were captured, eliminating selection bias.

\section{Conclusion}

We identified that, within the setting of provincially directed cancer care, there are regional differences in the care of patients with MIBC, specifically related to rates of NACT consultation. Strategies are needed to ensure that all patients who are candidates for such therapy receive a consultation with a medical oncologist. Multidisciplinary tumour boards promote the discussion of patients in a more comprehensive manner and may be one way to improve the rate of NACT consultations among patients with MIBC in Alberta.

Acknowledgements: The authors would like to thank Emily MacLeod (CancerControl Alberta) for assistance with data collection.
Competing interests: The authors declare no competing financial or personal interests.

This paper has been peer-reviewed.

\section{References}

1. Statistics 2014. Toronto, ON: Canadian Cancer Society. cancer.ca/statistics. Accessed June 15, 2015.

2. Statistics Canada. CANSIM Tables. http://cansim2.statcan.gc.ca. Accessed June 15, 2015.

3. Tilki D, Reich 0, Karakiewicz PI, et al. Validation of the AJCC TNM substaging of pT2 bladder cancer: Deep muscle invasion is associated with significantly worse outcome. Eur Urol 2010;58:112-7. http:// dx.doi.org/10.1016/i.eururo.2010.01.015

4. Lamm DL, Blumenstein BA, Crissman JD, et al. Maintenance bacillus Calmette-Guerin immunotherapy for recurrent TA, $\mathrm{Tl}$ and carcinoma in situ transitional cell carcinoma of the bladder: A randomized Southwest Oncology Group study. J Urol 2000;163:1124-9 http://dx.doi.org/10.1016/S00225347(05)67707-5

5. Taher AN, Kotb MH. Bone metastases in muscle-invasive bladder cancer. J Egypt Natl Canc Inst 2006;18:203-8.

6. Milowsky MI, Stadler WM, Bajorin DF. Integration of neoadjuvant and adjuvant chemotherapy and cystectomy in the treatment of muscle-invasive bladder cancer. BJU Int 2008;102:1339-44. http:// dx.doi.org/10.1111/i.1464-410X.2008.07980.x 
7. Gray PJ, Fedewa SA, Shipley WU, et al. Use of potentially curative therapies for muscle-invasive bladder cancer in the United States: Results from the National Cancer Data Base. Eur Urol 2013;63:823-9. http://dx.doi.org/10.1016/i.eururo.2012.11.015

8. Grossman $H B$, Natale RB, Tangen $C M$, et al. Neoadjuvant chemotherapy plus cystectomy compared with cystectomy alone for locally advanced bladder cancer. N Engl J Med 2003;349:859-66. http://dx.doi. org/10.1056/NEJMoa022148

9. International Collaboration of Trialists, the European Organisation for Research and Treatment of Cancer Genito-Urinary Tract Cancer Group, the Australian Bladder Cancer Study Group, et al. International Phase III Trial Assessing Neoadjuvant Cisplatin, Methotrexate, and Vinblastine Chemotherapy for Muscle-Invasive Bladder Cancer: Long-Term Results of the BA06 30894 Trial. J Clin Oncol 2011;29:2171-7. http:// dx.doi.org/10.1200/JC0.2010.32.3139

10. Advanced Bladder Cancer Meta-Analysis Collaboration. Neoadjuvant chemotherapy in invasive bladder cancer: Update of a systematic review and meta-analysis of individual patient data. Eur Urol 2005;48:2026. http://dx.doi.org/10.1016/i.eururo.2005.04.006

11. Alberta Genitourinary Tumour Team. Muscle invasive and locally advanced/metastatic bladder cancer. albertahealthservices.ca/hp/if-hp-cancer-guide-gu002-bladder.pdf. Accessed June 15, 2015.

12. National Comprehensive Cancer Network. Bladder cancer. 2014; version 2. nccn.org/professionals/ physician_gls/pdf/bladder.pdf. Accessed June 15, 2015.

13. Witjes JA, Compérat E, Cowan NC, et al. EAU guidelines on muscle-invasive and metastatic bladder cancer: Summary of the 2013 guidelines. Eur Urol 2014;65:778-92. http://dx.doi.org/10.1016/i. eururo.2013.11.046. Epub 2013 Dec 12.

14. Seah JA, Blais N, North S, et al. Neoadjuvant chemotherapy should be administered to fit patients with newly diagnosed, potentially resectable muscle-invasive urothelial cancer of the bladder (MIBC): A 2013 CAGMO Consensus Statement and Call for a Streamlined Referral Process. Can Urol Assoc J 2013;7:312-8. http://dx.doi.org/10.5489/cuaj.1506

15. Stein JP, Lieskovsky $G$, Cote $R$, et al. Radical cystectomy in the treatment of invasive bladder cancer: Long-term results in 1,054 patients. J Clin Oncol 2001;19:666-75.

16. Miles BJ, Fairey AS, Eliasziw $M$, et al. Referral and treatment rates of neoadjuvant chemotherapy in muscle-invasive bladder cancer before and after publication of a clinical practice guideline. Can Urol Assoc J 2010;4:263-7. http://dx.doi.org/10.5489/cuaj09134

17. Alberta Innovates Health Solutions. ARECCl: A pRoject Ethics Community Consensus Initiative. http:// www.aihealthsolutions.ca/outreach-learning/arecci-a-project-ethics-community-consensus-initiative/. Accessed June 15, 2015.

18. Kulkarni GS, Urbach DR, Austin PC, et al. Impact of provider volume on operative mortality after radical cystectomy in a publicly funded healthcare system. Can Urol Assoc J 2013;7:425-9. http://dx.doi. org/10.5489/cuai.361
19. Pal SK, Ruel NH, Wilson TG, et al. Retrospective analysis of clinical outcomes with neoadjuvant cisplatinbased regimens for muscle-invasive bladder cancer. Clin Genitourin Cancer 2012;10:246-50. httr:// dx.doi.org/10.1016/i.dgc.2012.08.004

20. Meeks JJ, Bellmunt J, Bochner BH, et al. A systematic review of neoadjuvant and adjuvant chemotherapy for muscle-invasive bladder cancer. Eur Urol 2012;62:523-33. http://dx.doi.org/10.1016/i. eururo.2012.05.048

21. Hershman D, Jacobson JS, McBride R, et al. Effectiveness of platinum-based chemotherapy among elderly patients with advanced ovarian cancer. Gynecol Oncol 2004;94:540-9. http://dx.doi.org/10.1016/i. ygyno.2004.04.022

22. Bamias A, Efstathiou E, Moulopoulos LA, et al. The outcome of elderly patients with advanced urothelial carcinoma after platinum-based combination chemotherapy. Ann Oncol 2005;16:307-13. http://dx.doi. org/10.1093/annonc/mdi039

23. Booth CM, Siemens DR, Peng Y, et al. Patterns of referral for peri-operative chemotherapy among patients with muscle-invasive bladder cancer (MIBC): A population-based study. J Clin Oncol 2014;32:e15507.

24. Reardon ZD, Patel SG, Zaid HB, et al. Trends in the use of perioperative chemotherapy for localized and locally advanced muscle-invasive bladder cancer: A sign of changing tides. Eur Urol 2015;67:165-70. http://dx.doi.org/10.1016/i.eururo.2014.01.009

25. Newman EA, Guest AB, Helvie MA, et al. Changes in surgical management resulting from case review at a breast cancer multidisciplinary tumor board. Cancer 2006;107:2346-51. http://dx.doi.org/10.1002/ cncr.22266

26. Bumm R, Feith $M$, Lordick $F$, et al. Impact of multidisciplinary tumor boards on diagnosis and treatment of esophageal cancer. European Surgery 2007;39:136-40. http://dx.doi.org/10.1007/s10353-0070333-5

27. Dash A, Pettus JA, Herr HW, et al. A role for neoadjuvant gemcitabine plus cisplatin in muscle-invasive urothelial carcinoma of the bladder. Cancer 2008;113:2471-7. http://dx.doi.org/10.1002/cncr.23848

28. May M, Fritsche HM, Brookman-May S, et al. Patients with bladder cancer in clinical stage T2: Survival benefit of downstaging in comparison to patients with confirmed muscle invasion in cystectomy specimens. Urologe A 2010;49:1508-15. http://dx.doi.org/10.1007/s00120-010-2424-3

29. McCabe JE, Jibawi A, Javle PM. Radical cystectomy: Defining the threshold for a surgeon to achieve optimum outcomes. Postgrad Med J 2007;83:556-60. http://dx.doi.org/10.1136/pgmi.2007.058214

Correspondence: Geoffrey T. Gotto, Southern Alberta Institute of Urology, Suite 6638 - 7007 14th Street SW-Calgary, AB T2V 1P9; drgotto@gmail.com 